\title{
High frequency acoustic excitations in ordered diblock copolymer studied by inelastic $x$-ray scattering
}

\author{
H. Kriegs \\ Max Planck Institute for Polymer Research, Ackermannweg 10, D-55128 Mainz, Germany \\ and Forschungszentrum Juelich, IFF, D-52428 Juelich, Germany \\ W. Steffen \\ Max Planck Institute for Polymer Research, Ackermannweg 10, D-55128 Mainz, Germany \\ G. Fytas \\ Max Planck Institute for Polymer Research, Ackermannweg 10, D-55128 Mainz, Germany \\ and FORTH, Institute of Electronic Structure and Laser, P.O. Box 1527, 71110 Heraklion, Crete, Greece \\ G. Monaco \\ ESRF, European Synchrotron Radiation Facility, F-38043 Grenoble, France \\ C. Dreyfus \\ PMC, Case Postale 77, UPMC, 4 Place Jussieu, 75005 Paris, France \\ P. Fragouli, M. Pitsikalis, and N. Hadjichristidis \\ Department of Chemistry, University of Athens, Athens 15701, Greece
}

(Received 5 February 2004; accepted 7 May 2004)

\begin{abstract}
The phonon propagation in lamellar nanostructures formed via self-assembling of short styrene- $b$-isoprene (SI) as well as of its more incompatible styrene- $b$-(ethylene-alt-propylene) (SEP) counterpart was studied by inelastic x-ray scattering. Irrespective of the physical state of the block copolymers, a single acoustic phonon was observed in SI (ordered and disordered) and SEP (ordered). At GHz frequencies, inelastic light scattering from the same samples revealed very small dispersion in the sound phase velocity but a short phonon lifetime. () 2004 American Institute of Physics. [DOI: 10.1063/1.1767520]
\end{abstract}

\section{INTRODUCTION}

The study of acoustic excitations in condensed matter is a rich field of solid state physics with technological interest, e.g., in diagnostics. The propagation of sound in disordered viscoelastic materials has been studied in the past by ultrasonic relaxation. Brillouin light scattering (LS) and more recently by inelastic x-ray scattering (IXS) (Ref. 1) to reveal relaxation mechanisms active in the $\mathrm{MHz}, \mathrm{GHz}$, and $\mathrm{THz}$ range, respectively. In all these cases, one acoustic phonon with frequency $\omega$ is observed at a given wave vector $q$ and its dispersion relationship $\omega$ vs $q$ or $\omega$ vs temperature at constant $q$ is usually studied. In inhomogeneous systems, with structure on the length scale of the order of $q^{-1}$, however, several acoustic excitations are possible resulting in a rich dispersion relation $\omega_{n}$ versus $q$. Morphological, geometrical, and micromechanical properties are manifested in the phonon spectrum of ordered mesoscopic soft structures, e.g., colloidal crystalline suspensions ${ }^{2}$ and lamellar ordered diblock copolymer solutions, ${ }^{3}$ studied by high resolution LS in the $\mathrm{GHz}$ range. In this context, the application of the latter technique requires structures with a spacing $d$ of a few hundred nanometers, i.e., $q d=O(1)$.

Soft nanostructures can be readily formed via selfassembling of low molecular mass diblock copolymers with strongly incompatible block components. These systems reveal one acoustic phonon when examined by LS with frequency $\omega=c q$ where $c$ is the phase velocity of sound in the effective medium. ${ }^{4}$ Alternatively, IXS possesses the necessary resolution $(\sim 1 / q)$ to probe displacements in the individual microphases well within the Brillouin zone. So far there are only few applications of IXS to amorphous or semicrystalline homopolymers ${ }^{5,6}$ but none addresses the ordered diblock copolymers. It is the objective of the present work to examine the potential of IXS to reveal the effect of nanostructure on the sound propagation in the $\mathrm{THz}$ range. Clearly, the sensitivity of the technique is enhanced in the presence of significant contrast in the elastic constants of the individual components. ${ }^{2}$ The high glass transition temperature $T_{g}$ in polystyrene $(\sim 370 \mathrm{~K})$ versus the low $T_{g}(\sim 210 \mathrm{~K})$ in polyolefins warrants an elastic constant contrast of about $30 \%$ with respect to each other in the intramolecular mixtures of these two components at ambient temperature.

The synthesis and molecular characteristics of a symmetric styrene-isoprene (SI) diblock with a lamellar spacing $d=17 \mathrm{~nm}$ is presented in the following section. To further enhance the block incompatibility, ${ }^{7}$ the lamellar ordered styrene- $b$-(ethylene-alt-propylene) (SEP) diblock ( $d$ $=20 \mathrm{~nm}$ ) was obtained via hydrogenation of the same parent SI as described in the Experiment. In Sec. III, IXS from both microphase separated SI and SEP reveals one single acoustic phonon for $q$ between 2 and $10 \mathrm{~nm}^{-1}$ (well beyond the first Brillouin zone at about $0.2 \mathrm{~nm}^{-1}$ ) with the high frequency speed of sound being very close to its value in the $\mathrm{GHz}$ range as measured by LS. In Sec. IV, we attribute this result to the 
TABLE I. Molecular characteristics of the diblock copolymers.

\begin{tabular}{lcccccc}
\hline \hline Sample & $\begin{array}{c}{ }^{\mathrm{a}} M_{n}\left(\times 10^{3}\right) \\
\text { PS block }\end{array}$ & $\begin{array}{c}{ }^{\mathrm{a}} M_{n}\left(\times 10^{3}\right) \\
\text { PS- } b-\mathrm{PI}\end{array}$ & $M_{w} / M_{n}$ & ${ }^{\mathrm{b}_{\mathrm{wt}} \%, \mathrm{PS}}$ & $T_{g}(\mathrm{~K})$ & $q^{*}\left(\mathrm{~mm}^{-1}\right)$ \\
\hline SI & 5.9 & 11.0 & 1.03 & $53.6(52.9)$ & 214,340 & 0.38 \\
SEP & 5.9 & 11.1 & 1.03 & $53.2(52.4)$ & 220,345 & 0.31 \\
\hline \hline
\end{tabular}

${ }^{a} \mathrm{By}$ SEC in THF at $40{ }^{\circ} \mathrm{C}$ using PS and PI standards and appropriate corrections.

${ }^{\mathrm{b}} \mathrm{By}{ }^{1} \mathrm{H}-\mathrm{NMR}$ spectroscopy in $\mathrm{CDCl}_{3}$ at $30{ }^{\circ} \mathrm{C}$ (calculated composition using the molecular weights of the individual blocks).

very short lifetime of the high $q$ phonon along with the moderate elastic constant contrast of the two microphases.

\section{EXPERIMENT}

\section{A. Synthesis}

The low molecular weight diblock copolymers SI were synthesized by sequential addition of styrene and isoprene to $s$-BuLi using well established high vacuum procedures. ${ }^{8}$ The polymers were hydrogenated in a Parr autoclave using the Wilkinson catalyst, ${ }^{9,10}$ prepared in situ by the reaction of $0.124 \mathrm{~g}$ of $\mathrm{RhCl}_{3} \cdot 3 \mathrm{H}_{2} \mathrm{O}$ and $0.73 \mathrm{~g}$ of $\mathrm{PPh}_{3}$ in tetrahydrofuran (THF) for $2 \mathrm{~h}$, at room temperature. The concentration of the polymer was $1.3 \% \mathrm{w} / \mathrm{v}$ and of the catalyst $120 \mathrm{ppm}$ in THF:toluene 1:1 w/v) solution. The reactor was flushed several times with argon and then several times with hydrogen. The reaction was allowed to take place at 35 bars and $373 \mathrm{~K}$ for $24 \mathrm{~h}$. The reactor was then cooled to room temperature and the hydrogenated polymer solution was extracted with an aqueous solution of $\mathrm{P}\left(\mathrm{O}_{6} \mathrm{H}_{4}-m-\mathrm{SO}_{3} \mathrm{Na}\right)_{3}$ to remove the catalyst. The organic phase was dried overnight using sodium sulfate, the polymer was precipitated in methanol, filtered and dried in a vacuum oven, until constant weight. The ${ }^{1} \mathrm{H}-\mathrm{NMR}$ analysis revealed that $63 \%$ of the I block was hydrogenated into ethylene-alt-propylene (EP) block, while no hydrogenation of the phenyl groups was detected.

\section{B. Characterization}

Size exclusion chromatography (SEC) experiments were conducted at $313 \mathrm{~K}$ using a modular instrument consisting of a Waters Model 510 pump, a Waters Model U6K sample injector, a Waters Model 401 differential refractometer, a Waters Model 486 UV spectrophotometer, and a set of $4 \mathrm{~m}$ Styragel columns with a continuous porosity range from $10^{6}$ to $10^{3} \AA$. THF was the carrier solvent at a flow rate of 1 $\mathrm{ml} / \mathrm{min}$. The calibration curve of the instrument was obtained by using nine polystyrene and nine polyisoprene standards with molecular weights ranging from 2000 up to 900000 . The ${ }^{1} \mathrm{H}$-NMR spectra of the polymers were obtained using a Bruker AC200 spectrometer at $303 \mathrm{~K}$ using $\mathrm{CDCl}_{3}$ as the solvent. The molecular characteristics of the PS precursors and the final model diblock copolymers, prepared by high vacuum techniques, are provided in Table I. The results clearly show that the samples have high molecular and compositional homogeneity.

The glass transition temperature $T_{g}$ was determined by differential scanning calorimetry at $10 \mathrm{~K} / \mathrm{min}$. Expectedly, the microphase separated samples exhibit two $T_{g}$ 's one for the soft (I or EP) block at low temperatures around $215 \mathrm{~K}$ and one for the hard (S) block at high temperatures around $340 \mathrm{~K}$ (Table I). The physical state and the characteristic spacing of the SI and SEP samples were obtained from small angle $\mathrm{x}$-ray scattering (SAXS) experiments performed with a rotating anode (Rigaku) as source and a two-dimensional (2D) detector. Based on the scattering intensity $I(q)$ profiles shown in Fig. 1 both samples are in the lamellar ordered state at ambient temperature. The SI sample is in the disordered state at $413 \mathrm{~K}$ while the SEP sample resides in the ordered state up to the highest examined temperature of 413 $\mathrm{K}$. The nearly absence of the second peak at $2 q^{*}$ in the $I(q)$ further indicates a well formed lamellar state for these compositionally symmetric diblock copolymers. The spacing $d$ $=2 \pi / q^{*}$ with $q^{*}$ (Table I) being the position of the main peak of $I(q)$ amounts to $17 \mathrm{~nm}$ for SI and $20 \mathrm{~nm}$ for the SEP due to the enhanced incompatibility between $S$ and EP blocks. ${ }^{7}$

\section{Inelastic x-ray scattering (IXS)}

For the IXS experiment the samples were melted under vacuum into cylindrical cells $6 \mathrm{~mm}$ in diameter and $13 \mathrm{~mm}$ long. The sample length was chosen to be comparable to the photoabsorption length at the x-ray beam energy (21.748 $\mathrm{keV})$. The cells were closed by windows made of mica (Goodfellow) of a thickness of $30( \pm 5) \mu \mathrm{m}$. The contribution of the mica windows was found to be negligible.

The experiment was carried out at the very high energy resolution IXS beamline ID 16 at the European Synchrotron Radiation Facility in Grenoble. The highly monochromatic incident beam was obtained by a combination of a cryogeni-

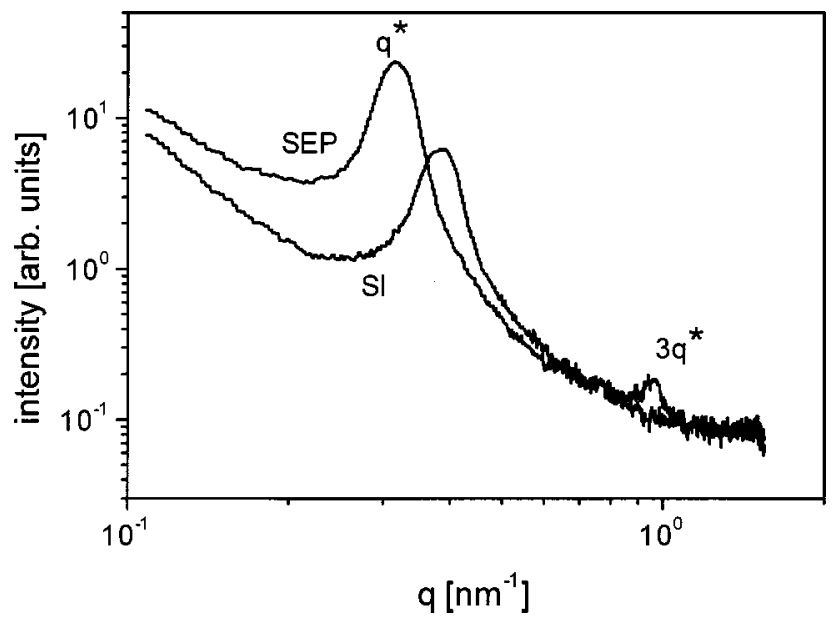

FIG. 1. Intensity distribution $I(q)$ in undiluted SEP and SI at $293 \mathrm{~K}$ in a SAXS experiment as indicated. 


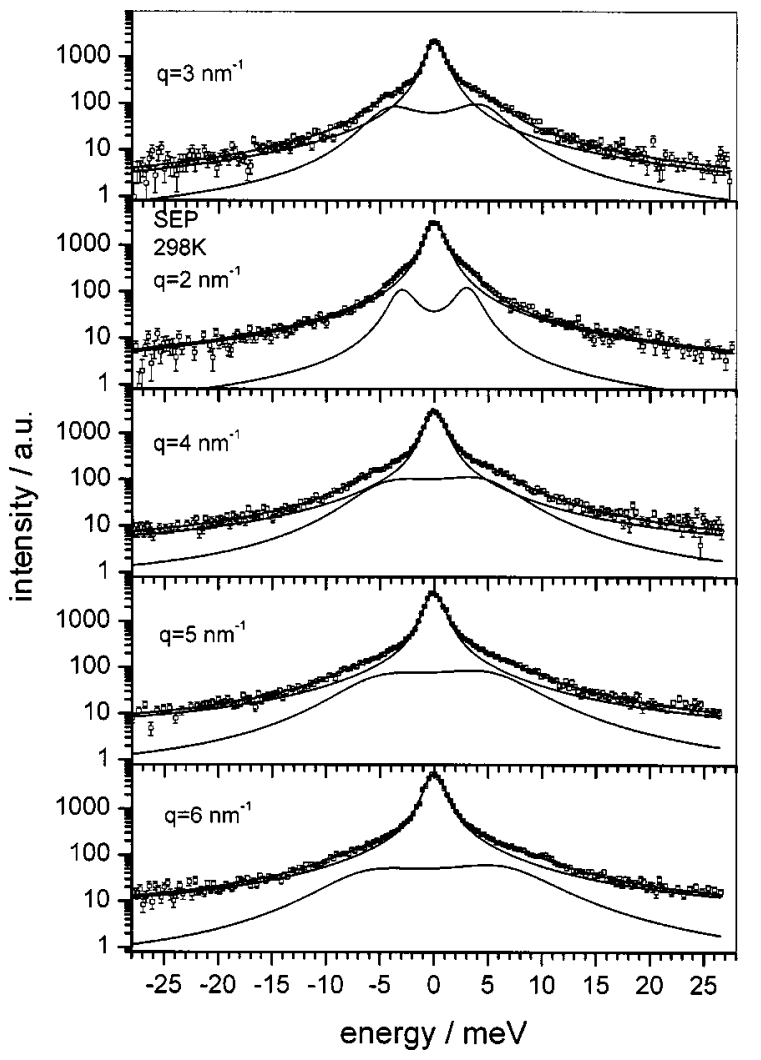

FIG. 2. Inelastic x-ray spectra of SEP in the lamellar ordered state at the indicated $q$ values at $298 \mathrm{~K}$. Lines indicate the central elastic, the inelastic (shifted peaks), and total contributions to the total spectrum as represented by Eq. (1)

cally cooled $\mathrm{Si}(111)$ double monochromator and a backscattering monochromator working at the $\mathrm{Si}(11$ 11 11) Bragg reflection. ${ }^{11}$ The beam was characterized by an energy of $21.478 \mathrm{keV}$ and an intensity of $2 \times 10^{9}$ photons/s at the sample stage. The $\mathrm{x}$ rays scattered from the sample were collected by spherical analyzers working as well as the monochromator in backscattering at the $\operatorname{Si}\left(\begin{array}{lll}11 & 11 & 11\end{array}\right)$ reflection. Five analyzers are available to collect spectra at five different momentum transfers, $q_{i}(i=1-5)$, at the same time; $q_{i}=4 \pi / \lambda \sin \left(\theta_{i} / 2\right)$ with $\lambda$ the incident wavelength and $\theta_{i}$ the scattering angle. The energy scans were performed by varying the relative temperature between the monochromator and the analyzer crystals. The total energy resolution amounts to $1.5 \mathrm{meV}$ full width at half maximum determined with a Plexiglass sample at a $q$ corresponding to the maximum of its static structure factor. The resolution in $q$ was $\pm 0.4 \mathrm{~nm}^{-1}$.

\section{RESULTS}

Figure 2 displays IXS spectra $I(q, \omega)$ of the lamellar ordered SEP at five different $q$ values at $298 \mathrm{~K}$. The spectra are represented by a sum of a central Lorentzian and of a damped harmonic oscillator (DHO) to describe the quasielastic and the inelastic spectral components, respectively,

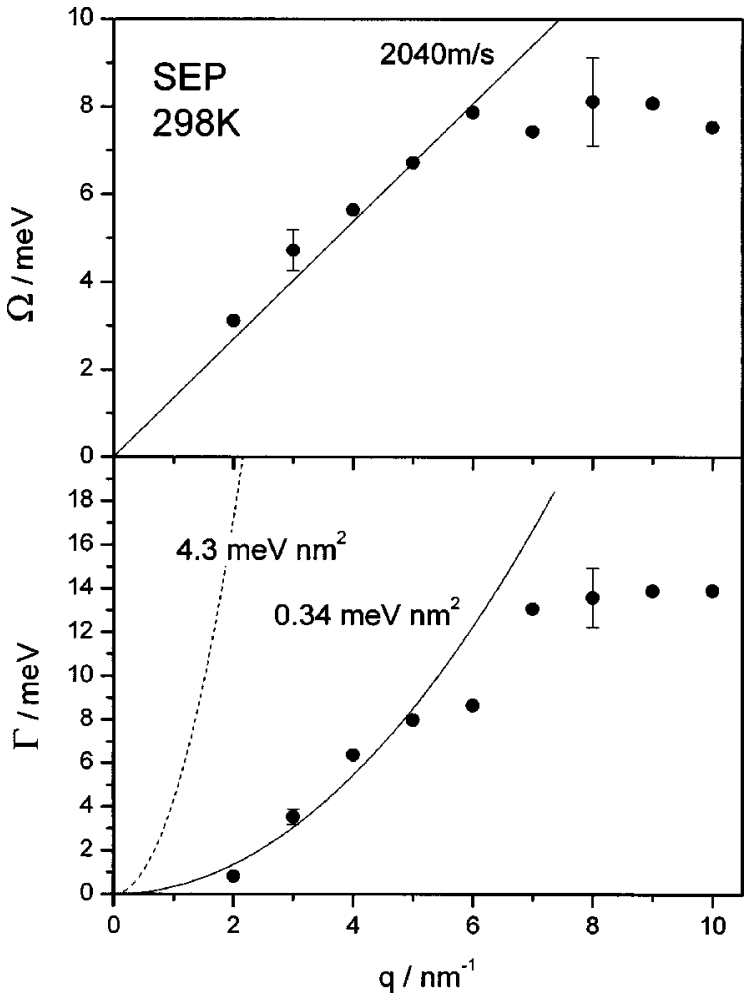

FIG. 3. (a) Dispersion relation $\Omega(q)$ vs $q$ in lamellar ordered SEP at $298 \mathrm{~K}$. The solid line indicates the acoustic phonon frequency using $c=2040 \mathrm{~m} / \mathrm{s}$ from the Brillouin light scattering experiment (Fig. 4). (b) The width of the IXS spectra obtained from their representation by Eq. (1). The solid and dashed lines denote the $q^{2}$ hydrodynamic dependence of the width in the IXS and LS experiments, respectively. Typical errors at low and high $q$ are indicated by the bars.

$$
I(q, \omega)=\frac{\alpha_{e} \Gamma_{e}}{\left(\omega^{2}+\Gamma_{e}^{2}\right)}+\frac{\alpha_{\mathrm{in}} \frac{\hbar \omega}{k_{B} T}[n(\omega)+1] \Gamma(q) \Omega^{2}(q)}{\left(\Omega^{2}(q)-\omega^{2}\right)^{2}+(\Gamma(q) \omega)^{2}} .
$$

Here, $[n(\omega)+1] \equiv 1 /\left[1-\exp \left(-\hbar \omega / k_{B} T\right)\right]$ accounts for the different population on the Stokes and anti-Stokes sides. This model function is able to represent well the experimental data, as shown in Fig. 2. The elastic and inelastic contributions to the total $I(q, \omega)$ are indicated by the central and shifted lines in each panel of Fig. 2. The frequency $\Omega(q)$ and the width $\Gamma(q)$ of the inelastic peaks (Fig. 3 for the case of SEP) systematically increase with $q$ while the relative inelastic amplitude $\alpha_{\text {in }} / \alpha_{e}$ is virtually $q$ independent. The dispersion relation $\Omega(q)$ versus $q$ shown in Fig. 3(a) is linear for $q<6 \mathrm{~nm}^{-1}$ and hence allows the estimation of the sound velocity $c_{\propto}=\Omega(q) / q$ at very high $(\mathrm{THz})$ frequencies.

The sound velocity can also be obtained at lower $\mathrm{GHz}$ frequencies from the LS spectra of Fig. 4. From the frequency shift of the single Brillouin doublet at $q$ $=0.025 \mathrm{~nm}^{-1}$, the phase velocity $c$ of the acoustic phonon amounts to $2170 \mathrm{~m} / \mathrm{s}$ in SI and $2040 \mathrm{~m} / \mathrm{s}$ in SEP at $293 \mathrm{~K}$. The slightly lower value of $c$ in the latter probably results from the stronger phonon dispersion in SEP due to the effect of the segmental relaxation. Despite the glassy S block, the broad Brillouin lines indicate fast local structural dynamics in the soft (I and EP) microphases. ${ }^{12}$ 


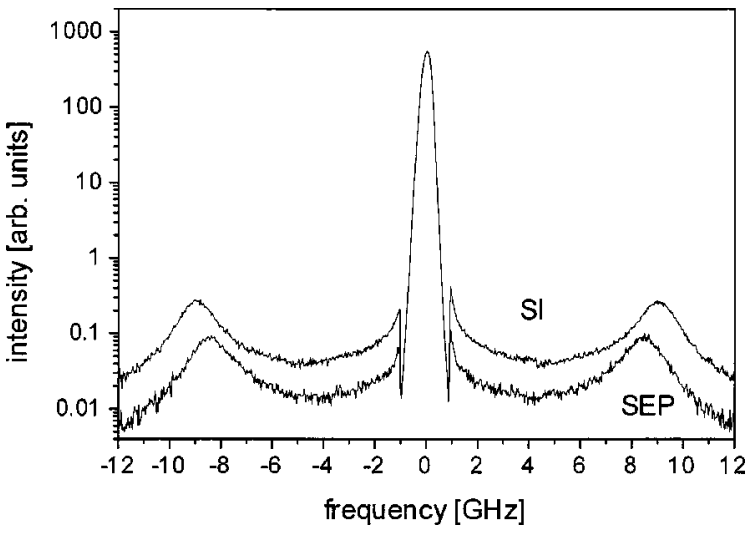

FIG. 4. Brillouin spectra of SEP and SI as indicated on the plot at $q$ $=0.025 \mathrm{~nm}^{-1}$ and $293 \mathrm{~K}$ recorded with a tandem Fabry-Perot interferometer. The frequency range $\pm 1 \mathrm{GHz}$ around the central line is reserved for the automatic stabilization of the interferometer using a reference laser beam.

The sound velocity $c_{\propto}$ is very close to $c=2040 \mathrm{~m} / \mathrm{s}$ of SEP obtained from LS at $293 \mathrm{~K}$ and the value of $c$ is used to draw the solid line in Fig. 3(a). For $q>6 \mathrm{~nm}^{-1}$, this acoustic excitation becomes more and more overdamped and progressively looses its propagative character. The dispersion curve in the microphase separated diblock copolymer of Fig. 3 and in amorphous polybutadiene, ${ }^{5}$ are qualitatively alike and resemble that of atomic systems. According to the dispersion relation in these systems, the deviation from the linear $\omega$ versus $q$ dependence above about $6 \mathrm{~nm}^{-1}$ corresponds to $\pi / a$ with $a=0.5 \mathrm{~nm}$ of the order of the segmental length.

The width $\Gamma(q)$ [Fig. 3(b)] of the inelastic peaks displays a nonlinear increase with $q$ conforming to the hydrodynamic $q^{2}$ dependence up to about $5 \mathrm{~nm}^{-1}$. The coefficient $\Gamma / q^{2} \sim 0.34 \mathrm{meV} \mathrm{nm}^{2}$, however, is clearly lower than the corresponding value of about $4.3 \mathrm{meV} \mathrm{nm}^{2}$ in the $\mathrm{GHz}$ range (Fig. 4). In spite of the vitrification of the S block (see high $T_{g}$ values in Table I), there is a significant line broadening in the LS spectra due to segmental relaxation in EP block (low $T_{g}$ component in Table I).

\section{DISCUSSION}

Brillouin light scattering has been previously employed ${ }^{4}$ to study the microphase separation in a low molecular mass asymmetric SI (S spheres with cubic symmetry). A single longitudinal long wavelength acoustic phonon is observed at low $q R=0.2$ (sphere radius of $7 \mathrm{~nm}$ ). For ultrahigh molar mass diblock copolymers $\left(M_{w} \approx 1-3 \times 10^{6} \mathrm{~g} / \mathrm{mol}\right)$, however, the same technique has revealed several phonons depending on the momentum transfer between photons, phonons, and lattice. ${ }^{3}$ A theoretical and structure analysis has captured the experimental dispersion relations for a lamellar morphology. For this 1D structure of spacing $d$, the reduced dispersion plot $f(q) d / c$ vs $q d / \pi$ in Fig. 5 can serve as a phonon mapping $(f=\Omega / 2 \pi)$. The theoretical dispersion relation in the inset of Fig. 5 was verified ${ }^{3}$ for lamellar ordered solutions of very high molecular weight (with $c=1380 \mathrm{~m} / \mathrm{s}$ and $d$ $\sim 200 \mathrm{~nm}$ ) by LS. For comparison, the present ordered SEP has $c=2040 \mathrm{~m} / \mathrm{s}$ and $d=17 \mathrm{~nm}$.

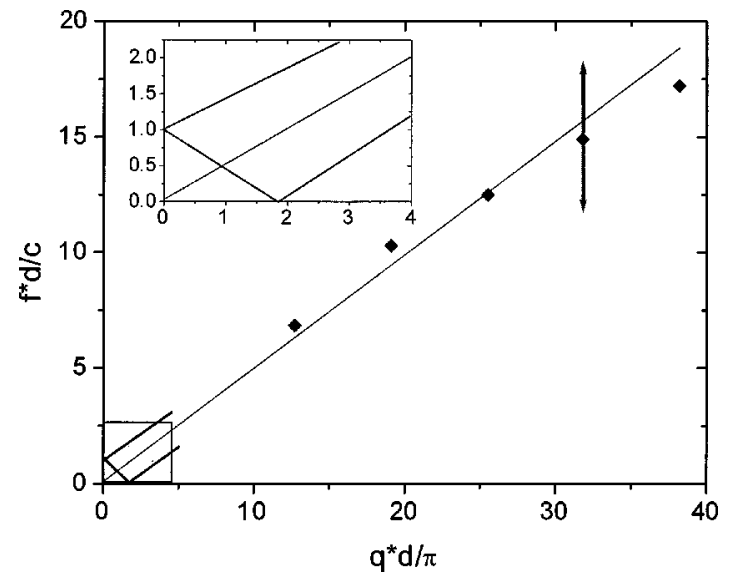

FIG. 5. Phonon dispersion relation in lamellar ordered diblock copolymers presented in the normalized $f d / c$ vs $q d$ plot ( $c$ is the sound velocity and $d$ the spacing). The symbols represent IXS data from Fig. 3(a) and the arrow indicates the linewidth at $q=51 \mathrm{~nm}^{-1}$ [Fig. 3(b)]. The inset as enlargement of the low $q d / \pi$ regime represents the calculated dispersion relations for a lamellar morphology (Ref. 3).

At $q d / \pi<4$-including the first and higher Brillouin zones-Fig. 5 and its enlarged inset show several phonons which can be related to the geometrical and morphological characteristics of the mesoscopic lamellar structure. ${ }^{13}$ On the contrary, IXS at much higher $q d$ values is found to arise only from the acoustic like phonon of the average medium over the whole $q$ range as shown in Fig. 5. Albeit the high spatial resolution of IXS and the fact that several individual mi-

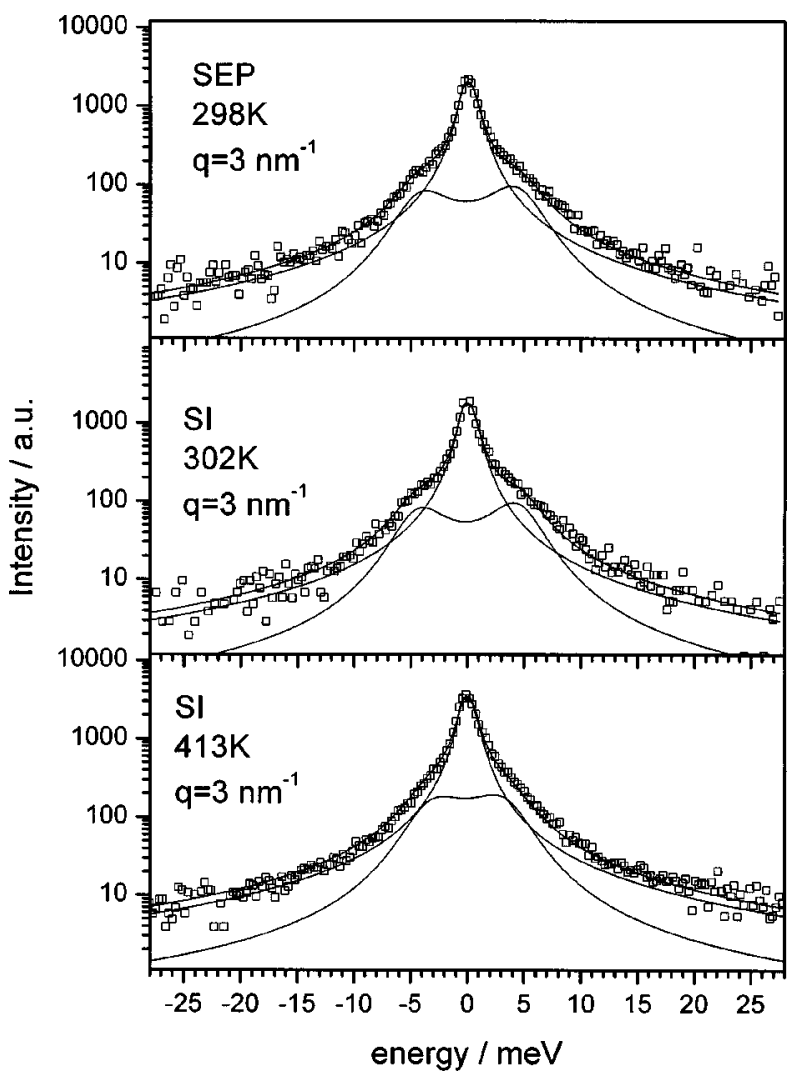

FIG. 6. IXS spectra of ordered SEP (a) and SI (b) at $3 \mathrm{~nm}^{-1}$ at $300 \mathrm{~K}$ and disordered SI at $3 \mathrm{~nm}^{-1}$ at $413 \mathrm{~K}$. The lines indicate the two contributions of Eq. (1) used in the fit to represent the experimental spectra. 
crophases are probed, nonetheless the system appears homogeneous for the sound propagation. In fact, the IXS spectrum of disordered (at $413 \mathrm{~K}$ ) and ordered (302 K) SI are qualitatively the same, as seen in Fig. 6 in comparison with the spectrum of the ordered SEP. The inelastic peaks in the IXS spectra are less pronounced at the high temperatures due to the decrease of sound velocity with decreasing temperature. A possible reason for the insensitivity of IXS to the physical state of diblock copolymer melts is the broad width and hence the short lifetime (about $\tau_{p}=0.2 \mathrm{ps}$ for $q=3 \mathrm{~nm}^{-1}$ ) of the acoustic phonon that decays within a distance of $0.4 \mathrm{~nm}$ ( $=c \tau_{p}$ ) which is even smaller than the spacing $d$.

The linewidth of the acoustic excitations as measured in the IXS experiment is narrower than anticipated from a $q^{2}$ dependence of the $\Gamma(q)$ at $\mathrm{GHz}$ frequencies. In the LS spectrum $\Gamma / q^{2} \cong 4.3 \mathrm{meV} \mathrm{nm}^{2}$ whereas in the $\mathrm{THz}$ regime this quantity amounts to $0.34 \mathrm{meV} \mathrm{nm}^{2}$ as shown in Fig. 3(b). In the high frequencies of the IXS the fast relaxation limit $\omega \tau \gg 1$, with $\tau$ being the characteristic relaxation time, is fulfilled and hence the sound dissipation due to the segmental relaxation in the mobile SEP is low. The latter is active in the $\mathrm{GHz}$ range and is responsible for the phonon lifetime (Fig. 4). A similar behavior was observed in the case of the low molecular weight glass forming liquid $o$-terphenyl. ${ }^{13}$

In summary, IXS from nanostructures of short diblock copolymer chains with incompatible blocks yields a single acoustic phonon of the average medium. The individual phases with an elastic contrast of about $30 \%$ appear homo- geneous for the phonon propagation at $\mathrm{THz}$ frequencies probably due to the high $q d$ values in connection with the short phonon lifetime. At present, inelastic light scattering is better suited for ordered structures with mesoscopic length scales.

${ }^{1}$ G. Monaco, A. Cunsolo, G. Ruocco, and F. Sette, Phys. Rev. E 60, 5505 (1999).

${ }^{2}$ R. S. Penciu, H. Kriegs, G. Petekidis, E. N. Economou, and F. Fytas, J. Chem. Phys. 118, 5224 (2003); R. S. Penciu et al., Europhys. Lett. 58, 693 (2002).

${ }^{3}$ A. Urbas, E. L. Thomas, H. Kriegs, G. Fytas, R. Penciu, and E. N. Economou, Phys. Rev. Lett. 90, 108302 (2003); G. Tommaseo, R. S. Penciu, G. Fytas, E. N. Economou, T. Hashimoto, and N. Hadjichristidis, Macromolecules (in press).

${ }^{4}$ G. Floudas, W. Steffen, and N. Hadjichristidis, Europhys. Lett. 44, 37 (1998).

${ }^{5}$ D. Fioretto et al., Phys. Rev. E 59, 4470 (1999).

${ }^{6}$ A. Mermet, L. David, M. Lorenzen, and M. Krisch, J. Chem. Phys. 119, 1879 (2003)

${ }^{7}$ C. Lai, W. B. Russel, R. A. Register, G. R. Marchand, and D. H. Adamson, Macromolecules 33, 3461 (2000); P. Holmquist, S. Pispas, N. Hadjichristidis, G. Fytas, and R. Sigel, ibid. 36, 830 (2003).

${ }^{8}$ N. Hadjichristidis, H. Iatrou, S. Pispas, and M. Pitsikalis, J. Polym. Sci., Part A: Polym. Chem. 38, 3211 (2000).

${ }^{9}$ H. Schmalz, A. Boker, R. Lange, and V. Abetz, Polym. Mater. Sci. Eng. 85, 478 (2001).

${ }^{10}$ N. A. Mohammadi and G. L. Rempel, J. Mol. Catal. A: Chem. 135, 121 (1998).

${ }^{11}$ R. Verbeni et al., J. Synchrotron Radiat. 3, 62 (1996).

${ }^{12}$ J. Kanetakis, G. Fytas, and N. Hadjichristidis, Macromolecules 24, 1806 (1991).

${ }^{13}$ G. Monaco, D. Fioretto, C. Masciovecchio, G. Ruocco, and F. Sette, Phys. Rev. Lett. 82, 1776 (1999). 